medRxiv preprint doi: https://doi.org/10.1101/2021.07.28.21261262; this version posted July 31, 2021. The copyright holder for this preprint (which was not certified by peer review) is the author/funder, who has granted medRxiv a license to display the preprint in perpetuity. It is made available under a CC-BY-NC-ND 4.0 International license .

\title{
Motivators and barriers to research participation for individuals with cerebral palsy and their families
}

${ }^{*}$ Kristina M. Zvolanek 1,2 , BS, *Vatsala Goyal'1,2, MS, Alexandra Hruby ${ }^{1,2}$, BS, Carson Ingo ${ }^{1,3}, \mathrm{PhD}$, Theresa Sukal-Moulton ${ }^{1,4}, \mathrm{PT}, \mathrm{DPT}, \mathrm{PhD}$

${ }^{*}$ Contributed equally as co-first authors

Short Title: Motivators and barriers to cerebral palsy research participation

\section{Affiliations:}

${ }^{1}$ Department of Physical Therapy \& Human Movement Sciences, Feinberg School of Medicine, Northwestern University, Chicago, IL, USA

${ }^{2}$ Department of Biomedical Engineering, Northwestern University, Evanston, IL, USA

${ }^{3}$ Department of Neurology, Feinberg School of Medicine, Northwestern University, Chicago, IL, USA

${ }^{4}$ Department of Pediatrics, Feinberg School of Medicine, Northwestern University, Chicago, IL, USA

\section{Corresponding Author:}

Theresa Sukal-Moulton, PT, DPT, PhD

645 N Michigan Ave Suite 1100, Chicago, IL 60611

Phone: (312) 503-3342

Email: theresa-moulton@northwestern.edu

Conflicts of Interest: There are no conflicts of interest.

Keywords: Cerebral palsy, research participation, survey, stakeholder attitudes

\section{Abbreviations:}

- CP - cerebral palsy

- GMFCS - Gross Motor Function Classification System

- REDcap - Research Electronic Data Capture 
medRxiv preprint doi: https://doi.org/10.1101/2021.07.28.21261262; this version posted July 31, 2021. The copyright holder for this preprint (which was not certified by peer review) is the author/funder, who has granted medRxiv a license to display the preprint in perpetuity. It is made available under a CC-BY-NC-ND 4.0 International license .

\begin{abstract}
Objective(s): Our objective was to investigate the motivators and barriers associated with the individual or family decision to participate in cerebral palsy research. Based on this information, we offer suggestions to increase the likelihood of participation in future CP studies.
\end{abstract}

Methods: A digital survey was administered to stakeholders affected by cerebral palsy across the US. Our analysis focused on variables related to personal interests, travel, and study-specific elements. Statistical tests investigated the effects of responder type, cerebral palsy type, and Gross Motor Function Classification System level on travel and study-specific element variables. Recommendations were informed by responses reflecting the majority of respondents.

Results: Based on 233 responses, we found that respondents highly valued research participation (on average 88.2/100) and compensation (on average 62.3/100). Motivators included the potential for direct benefit (62.2\%) and helping others (53.4\%). The primary barriers to participation were schedule limitations (48.9\%) and travel logistics (32.6\%). Schedule limitations were especially pertinent to caregivers, while individuals with more severe cerebral palsy diagnoses reported the necessity of additional items to comfortably travel.

Conclusions: Overall, we encourage the involvement of stakeholders affected by cerebral palsy in the research process. Researchers should consider offering flexible study times, accommodating locations, and compensation for time and travel expenses. We recommend a minimum compensation of $\$ 15 /$ hour and a maximum time commitment of 4 hours/day to respect participants' time and increase likelihood of research participation. Future studies should track how attitudes toward research change with time and experience. 
medRxiv preprint doi: https://doi.org/10.1101/2021.07.28.21261262; this version posted July 31, 2021. The copyright holder for this preprint (which was not certified by peer review) is the author/funder, who has granted medRxiv a license to display the preprint in perpetuity. It is made available under a CC-BY-NC-ND 4.0 International license .

\section{Introduction}

Cerebral palsy $(\mathrm{CP})$ is a broad pediatric-onset diagnosis caused by a nonprogressive injury to the developing brain [1]. The etiology of CP is also extremely heterogeneous, often resulting from brain injuries that occur during the early developmental period [2-5]. Although CP is considered a pediatric-onset disorder, the associated physical and behavioral presentations are present across the lifespan and may fluctuate in severity over time. As there is currently no cure for $\mathrm{CP}$, research efforts are critical for advancing our understanding of the pathophysiology and most efficacious treatments. However, the diversity of this population poses a significant recruitment challenge to researchers [6]. Limited funding for CP research also puts an added burden on researchers to be efficient with study-specific elements and recruitment, especially in the United States [7]. In an attempt to address this, previous studies have surveyed individuals with CP to identify priority research areas [8-10]. Research registries have also been established to facilitate collaboration among US institutions and to improve communication between researchers and individuals with CP $[11,12]$. Despite these efforts, the success of CP research is dependent on the desire of individuals to participate and their ability to reasonably access the study within the limitations of their environment.

A previous study investigated the barriers to intervention-based CP research and recommended the involvement of patient populations and their families in the study development pipeline to improve recruitment [6], an approach towards community-based participatory research. However, the study did not consider facilitators to research and only evaluated a small subset of the population interested in home-based training programs. Therefore, our objective was to sample a larger and more heterogeneous 
medRxiv preprint doi: https://doi.org/10.1101/2021.07.28.21261262; this version posted July 31, 2021. The copyright holder for this preprint (which was not certified by peer review) is the author/funder, who has granted medRxiv a license to display the preprint in perpetuity. It is made available under a CC-BY-NC-ND 4.0 International license .

cohort of stakeholders and investigate the motivators and barriers associated with the decision to participate in CP research. We aimed to inform researchers of specific stakeholder perspectives by understanding whether factors such as Gross Motor Function Classification System (GMFCS) level, CP type, or age contribute to the decision to participate. Based on a nationwide survey, we provide recommendations for investigators to increase likelihood of recruitment and participation in future CP research.

\section{Methods}

\section{Recruitment}

The survey, including informed consent, was created and administered using the Research Electronic Data Capture (REDCap) platform. It was approved by the Northwestern University Institutional Review Board and remained open between May 6th and July 7th, 2020. Respondents were eligible to voluntarily participate if they resided in the United States and were either 1) caregivers of minors (under 18 years of age) with a diagnosis of CP or 2) adults with a diagnosis of CP. The survey link was shared via several platforms, including the Cerebral Palsy Research Registry [11], ResearchMatch.org, department social media accounts, and emails to previous research participants.

\section{Experimental Protocol: Qualitative Survey}

The digital open survey was designed to collect data about motivators and barriers of participation in CP research. During survey development, we sought feedback from caregivers of minors with $\mathrm{CP}$ and adults with $\mathrm{CP}$ on clarity of questions and importance/completeness of content. The online survey (available in supporting 
medRxiv preprint doi: https://doi.org/10.1101/2021.07.28.21261262; this version posted July 31, 2021. The copyright holder for this preprint (which was not certified by peer review) is the author/funder, who has granted medRxiv a license to display the preprint in perpetuity. It is made available under a CC-BY-NC-ND 4.0 International license .

information) contained optional questions in six different categories: demographics, personal interests, travel needs \& preferences, study-specific elements, past research experience, and impact of COVID-19. The present analysis focused on the first four categories to summarize attitudes towards general CP research. Details of the survey development are described in further detail by Joshi et al. [13].

\section{Survey Categories}

\section{Demographics}

We collected a number of variables to describe features of the respondents, listed in Table 1. In addition to CP type and GMFCS level, we asked about elements such as the time it takes for respondents to get to medical appointments and information about current and previous medical treatments common to study inclusion or exclusion criteria.

\section{Personal Interests}

All subsequent variable names are italicized in text and described in Table 2. Personal interest factors were considered intrinsic to the respondent. Respondents were asked about their perception of research importance, how highly they value research participation, and compensation importance, all on a scale of 0-100. Open-ended questions in the survey requested comment on personal goals, motivators, and barriers for participation in $\mathrm{CP}$ research. To gauge specific research interests, respondents were asked what study types and body functions were of high interest to them. 
medRxiv preprint doi: https://doi.org/10.1101/2021.07.28.21261262; this version posted July 31, 2021. The copyright holder for this preprint (which was not certified by peer review) is the author/funder, who has granted medRxiv a license to display the preprint in perpetuity. It is made available under a CC-BY-NC-ND 4.0 International license .

\section{Travel Needs \& Preferences}

Respondents reported whether additional arrangements for childcare would be required, whether time off work would be required, and what additional travel needs would be required when leaving the house. To understand travel preferences, respondents were asked about their typical mode of transportation to medical appointments. We also asked participants to identify their perceived maximum travel time for indirect benefit study, maximum travel time for direct benefit study, and overall willingness to make an extended overnight trip for research participation. The importance of travel reimbursement for local study and travel reimbursement for distant study was also evaluated, both on a scale from $0-100$.

\section{Study-specific Elements}

There were a number of variables related to explicit design of the study, which have the potential to be modified by the researcher. Respondents were asked about their most preferred study locations and their preferred time of year for study participation. Respondents' desired compensation amount was evaluated per hour of study participation. Respondents were also asked about the maximum time commitment that was reasonable for one day of participation, the maximum study visits they would be willing to commit, and whether they would consider participating in a longitudinal study.

\section{Data and Statistical Analysis}

IBM SPSS Statistics version 26 (IBM Corp., Armonk, NY, USA) was used to perform all analyses on the survey responses. Participants with missing data for a given question were excluded from analysis pertaining to that question. To summarize 
medRxiv preprint doi: https://doi.org/10.1101/2021.07.28.21261262; this version posted July 31, 2021. The copyright holder for this preprint (which was not certified by peer review) is the author/funder, who has granted medRxiv a license to display the preprint in perpetuity. It is made available under a CC-BY-NC-ND 4.0 International license .

responses, descriptive analyses were first completed, with all percentages reported relative to the number of respondents for each question. We defined $50 \%$ as the threshold to describe the majority of survey respondents. Statistical analyses were only performed on travel and study-specific variables, as researchers can directly use this information to modify study methods during the developmental pipeline. Q-Q plots were created for quantitative variables to assess normality. Kruskal-Wallis tests, Chi-squared tests, or Kaplan-Meier survival analyses were performed on variables hypothesized to be dependent on three factors: Responder type (2 levels: adult, caregiver), CP type (4 levels: hemiplegia, diplegia, quadriplegia, other), and GMFCS level (5 levels: I, II, III, IV, V). A pvalue $<0.05$ was considered significant. Post hoc analyses were used to determine significant pairwise comparisons, where $p$-values were corrected for multiple comparisons using Bonferroni corrections. Further analyses were run to test specific hypotheses. For open-ended questions pertaining to personal interests, AH reviewed all responses, identified common themes, and categorized each response accordingly. Categorizations were reviewed and approved by KMZ and VG and summarized semiquantitatively. For travel preferences, Wilcoxon signed-rank tests were run to determine differences between travel time for indirect benefit and travel time for direct benefit and between importance of travel reimbursement for local study and travel reimbursement for distant study. 


\section{$\underline{\text { Results }}$}

\section{Demographics}

In total, 255 individuals were consented and 233 at least partially completed the survey. Respondent demographics are listed in Table 1. The survey population closely matches US census data in terms of sex, ethnicity, and race [13-15]. The majority of participants reported no previous research experience (53.9\%), a proximity to medical appointments of 1 hour or less (75.1\%), and previous or ongoing physical/occupational therapy treatment for their arms $(53.2 \%)$ or legs $(65.7 \%)$.

\section{Table 1: Participant demographics}

\begin{tabular}{|c|c|c|c|}
\hline \multirow[b]{2}{*}{ Characteristic } & \multicolumn{3}{|c|}{ Respondent } \\
\hline & $\begin{array}{c}\text { All } \\
(n=233)\end{array}$ & $\begin{array}{l}\text { Adult with } \\
\mathrm{CP}(\mathrm{n}=92)\end{array}$ & $\begin{array}{c}\text { Parent of } \\
\text { minor with } \\
\mathrm{CP}^{*}(n=141)\end{array}$ \\
\hline \multicolumn{4}{|l|}{ Sex assigned at birth } \\
\hline Male & 112 & 31 & 81 \\
\hline Female & 120 & 61 & 59 \\
\hline Not Reported & 1 & 0 & 1 \\
\hline \multicolumn{4}{|l|}{ Ethnicity } \\
\hline Hispanic or Latino & 23 & 7 & 16 \\
\hline Not Hispanic or Latino & 204 & 81 & 123 \\
\hline Not Reported & 6 & 4 & 2 \\
\hline \multicolumn{4}{|l|}{ Race } \\
\hline American Indian or Alaskan Native & 2 & 1 & 1 \\
\hline Asian & 9 & 3 & 6 \\
\hline Black or African American & 24 & 10 & 14 \\
\hline Native Hawaiian or Other Pacific Islander & 1 & 0 & 1 \\
\hline White & 175 & 68 & 107 \\
\hline Two or More Races & 6 & 4 & 2 \\
\hline Not Reported & 16 & 6 & 10 \\
\hline \multicolumn{4}{|l|}{ Gross Motor Function Classification System } \\
\hline Level I & 60 & 15 & 45 \\
\hline Level II & 65 & 33 & 32 \\
\hline Level III & 33 & 23 & 10 \\
\hline Level IV & 37 & 16 & 21 \\
\hline Level V & 35 & 4 & 31 \\
\hline Not Reported & 3 & 1 & 2 \\
\hline \multicolumn{4}{|l|}{ Cerebral Palsy motor type } \\
\hline Hemiplegia & 72 & 18 & 54 \\
\hline Diplegia & 60 & 37 & 23 \\
\hline Quadriplegia & 76 & 24 & 52 \\
\hline Other & 19 & 10 & 9 \\
\hline Not Reported & 6 & 3 & 3 \\
\hline \multicolumn{4}{|l|}{ Previous research experience } \\
\hline Yes & 101 & 40 & 61 \\
\hline
\end{tabular}




\begin{tabular}{|lccc|} 
No & 118 & 48 & 70 \\
Not Reported & 14 & 4 & 10 \\
Proximity to medical appointments & 58 & 24 & 34 \\
Less than 30 minutes & 108 & 45 & 63 \\
30 minutes to 1 hour & 55 & 19 & 36 \\
More than 1 hour & 12 & 8 & 4 \\
Not Reported & \multicolumn{3}{c|}{ Body Area } \\
\hline \multicolumn{1}{|c|}{ Medical Treatments Received } & Lrms & 57 & 11 \\
\hline Bony surgery & 4 & 109 & 1 \\
Soft tissue surgery & 13 & 6 & 6 \\
Neural surgery & 1 & 72 & 31 \\
Botox or other injections & 39 & 59 & 81 \\
Non-injectable spasticity medication & 35 & 153 & 20 \\
Physical or occupational therapy (current) & 124 & 39 & Spine \\
Intensive therapy programs/camps (previous) & 42 & & \\
\hline
\end{tabular}

*these demographics refer to the minor with cerebral palsy

\section{Personal Interests}

All subsequent variables and associated p-values are listed in Table 2. Respondents reported high mean scores for research importance (93.8/100), value research participation (88.2/100), and compensation importance (62.3/100). Open-ended questions revealed that the biggest personal motivators for $\mathrm{CP}$ research were personal benefit ( $62.2 \%$ of respondents) and helping others (53.4\%) (Figure $1 \mathrm{~A})$, while the biggest personal barrier was schedule limitations (48.9\%) (Figure 1B). With regard to research interests, the most popular study types were physical or occupational therapy treatments (90.8\%), activity monitoring (79.1\%), imaging of muscle/bone $(72.4 \%)$, survey or online $(71.5 \%)$, robotic games $(68.9 \%)$, imaging of the brain $(68.4 \%)$, and new treatments (64.9\%). The most popular body functions of interest were the legs/feet $(79.9 \%)$, muscles (79.5\%), movement/fitness (79.0\%), brain/nerves (76.0\%), arms/hands (62.9\%), and pain (50.7\%) (Figure 2). 
medRxiv preprint doi: https://doi.org/10.1101/2021.07.28.21261262; this version posted July 31, 2021. The copyright holder for this preprint (which was not certified by peer review) is the author/funder, who has granted medRxiv a license to display the preprint in perpetuity.
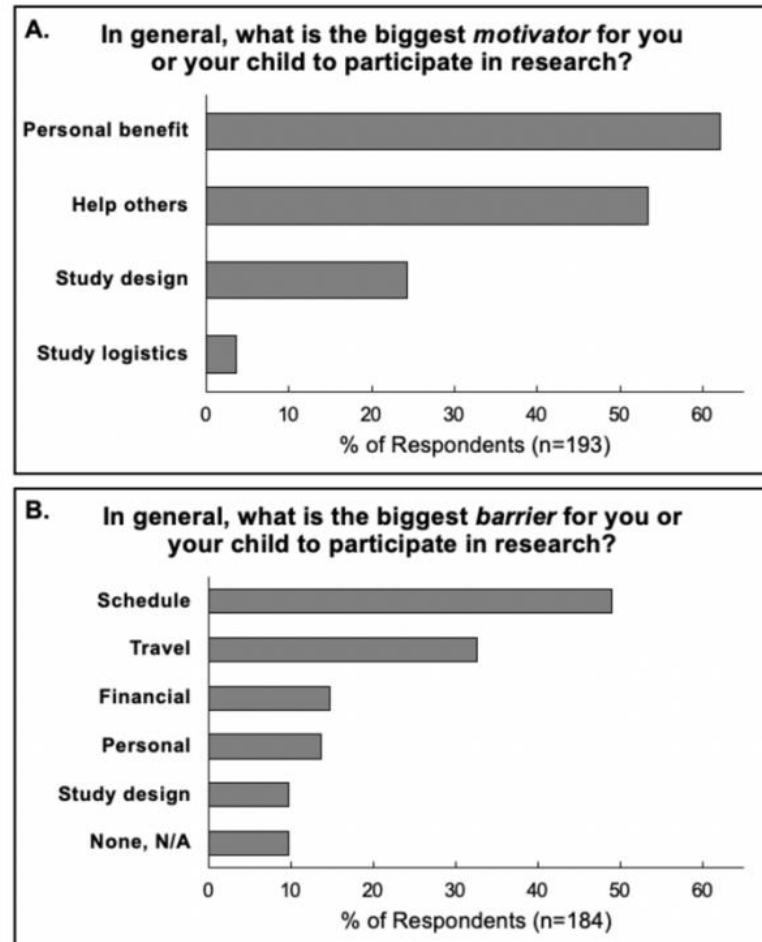

Personal benefit ( $\mathrm{n}=120)$ directly benefitting from participating Health benefit $(n=90)$ wanting better mobility, less pain, etc. for self or child; benefit related to condition

Education ( $\mathbf{n = 3 4}$ ) wanting to know more about their or their child's condition

Financial benefit ( $\mathbf{n = 1 2}$ ) compensation for participating Social benefit ( $n=7$ ) building a community, meeting new people Help others $(n=103)$ wanting to help others with $C P$

Research $(n=66)$ contribute to understanding/research of $C P$; find new treatments

Study design ( $\mathrm{n}=47$ ) study type or topic, length of study Study logistics ( $n=7$ ) convenience; time, location, date of study

Schedule limitations $(\mathbf{n = 9 0})$ amount of time, needing to plan around other obligations

Work $(\mathbf{n}=\mathbf{2 6})$ time off, scheduling around work responsibilities School $(n=16)$ not wanting child to miss school

Childcare $(n=11)$ needing to find and/or schedule childcare Travel limitations $(\mathbf{n}=60$ ) limitations related to getting to the study Location $(n=32)$ distance to research facility

Financial limitations $(\mathbf{n}=\mathbf{2 7})$ limitations related to cost of participating (transportation, food when at location, etc.)

Personal limitations $(\mathbf{n = 2 5})$ limitations related to participant's health Study design $(n=18)$ exclusion criteria, interest, expectations None, N/A $(n=18)$

c.

What would you hope to get out of your/your child's participation in research?

"Education, support, guidance, and funding to meet the individual needs of people who have CP." -Adult with GMFCS Level II CP

"I hope that I would be able to live a better life overall and hopefully some of my issues and pains would be improved through these studies." -Adult with GMFCS Level III quadriplegia

"I would love to be a part of research first and foremost that can be beneficial to him - I love helping with research for further studies and assistance for others but if we can get a benefit out of it, or potentially, that's even more motivation to participate. Anything that provides insight into his diagnosis is a benefit as well such as tests or MRIs etc." -Parent of child with GMFCS Level III CP

"Ideally, direct benefit, but I'd also be satisfied simply knowing that my participation helped advance the scientific community's understanding of CP and the people who live with it." -Adult with GMFCS Level II diplegia

Figure 1: Percentage of respondents who indicated $(A)$ motivators and $(B)$ barriers for participating in research relating to the categories shown. Indented categories are subcategories of the parent category. (C) Representative quotes indicating goals for participating in research. 


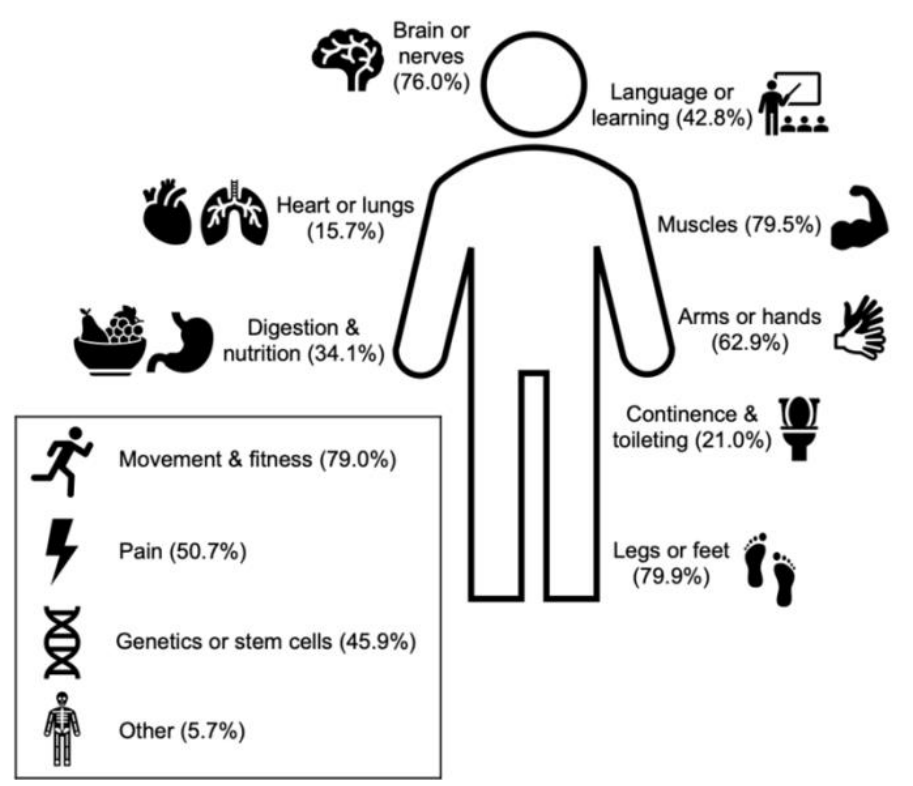

Figure 2: Body functions of research interest to respondents. Each of these options was offered as a checkbox for respondents to indicate if they would be interested in participating in a study that focused on these body regions/functions. Percentages are out of $n=233$ respondents.

Table 2: Summary metrics and statistical test results

\begin{tabular}{|c|c|c|c|c|c|}
\hline Variable & $\mathbf{N}$ & $\begin{array}{l}\text { Summary } \\
\text { Metrics } \\
\text { Median (IQR) } \\
\text { Mean (SD) }\end{array}$ & $\begin{array}{l}\text { GMFCS } \\
\text { Level }\end{array}$ & CP Type & $\begin{array}{l}\text { Responder } \\
\text { Type }\end{array}$ \\
\hline \multicolumn{6}{|l|}{ Personal Interest } \\
\hline $\begin{array}{l}\text { Research importance: } \\
\text { Importance of CP research (0-100) }\end{array}$ & 229 & $\begin{array}{c}99(9) \\
93.8(9.84)\end{array}$ & & & \\
\hline $\begin{array}{l}\text { Value research participation: } \\
\text { Value of participation in CP } \\
\text { research }(0-100)\end{array}$ & 221 & $\begin{array}{c}97(18) \\
88.2(17.7)\end{array}$ & & & \\
\hline $\begin{array}{l}\text { Compensation importance: } \\
\text { Importance of compensation for } \\
\text { study participation }(0-100)\end{array}$ & 203 & $\begin{array}{c}64(29) \\
62.3(25.7)\end{array}$ & & & \\
\hline $\begin{array}{l}\text { Study types: Study types most } \\
\text { likely to contact a researcher to } \\
\text { learn more about }\end{array}$ & 228 & $\begin{array}{l}\text { See Results: } \\
\text { Personal } \\
\text { Interests }\end{array}$ & & & \\
\hline $\begin{array}{l}\text { Body functions: Area of research } \\
\text { focus most interested in } \\
\text { participating or hearing more about }\end{array}$ & 233 & See Figure $2 A$ & & & \\
\hline
\end{tabular}




\section{Travel Needs \& Preferences}

Childcare: Whether additional

childcare is needed to participate

Time off work: Whether time off work is needed to participate

Additional travel needs: What other things need to be considered to travel to a research study

Time

Breathing

Transition

Seizure

Feeding

Snacks

Medications

Toileting

Transportation

Other

Transportation: Preferred transportation method

Drive self

Family member drives

Public transit

Ride service
221

220

174

Yes

Yes: 75

No: 146

$\chi^{2}(4)=4.27$ $\mathrm{p}=0.37$

$\chi^{2}(3)=6.88$, $\mathrm{p}=0.08$

$\chi^{2}(1)=24.0$,

Yes: 138

No: 82

$\chi^{2}(4)=6.29$,

$\mathrm{p}=0.18$

$\chi^{2}(3)=3.01$,

$\mathrm{p}=0.39$

$\chi^{2}(1)=1.04$, $\mathrm{p}=0.31$ $\mathbf{p}<0.001$

\section{See Figure $2 B$}

$$
\begin{aligned}
& \begin{array}{lll}
\chi^{2}(4)=11.7, & \chi^{2}(3)=14.0, & \chi^{2}(1)=0.012, \\
\mathrm{p}=0.02 & \mathbf{p}=0.003 & \mathrm{p}=0.91
\end{array} \\
& \chi^{2}(4)=17.4, \quad \chi^{2}(3)=2.76, \quad \chi^{2}(1)=0.146, \\
& \mathbf{p}=\mathbf{0 . 0 0 2} \quad \mathrm{p}=0.43 \quad \mathrm{p}=0.70 \\
& \chi^{2}(4)=12.4, \quad \chi^{2}(3)=4.12, \quad \chi^{2}(1)=6.87 \text {, } \\
& \mathrm{p}=0.02 \quad \mathrm{p}=0.25 \quad \mathrm{p}=0.009 \\
& \chi^{2}(4)=18.3, \quad \chi^{2}(3)=3.69, \quad \chi^{2}(1)=9.20, \\
& \mathbf{p}=\mathbf{0 . 0 0 1} \quad \mathrm{p}=0.30 \quad \mathbf{p}=\mathbf{0 . 0 0 2} \\
& \chi^{2}(4)=65.8, \quad \chi^{2}(3)=29.9, \quad \chi^{2}(1)=13.4 \text {, } \\
& \begin{array}{lll}
\mathbf{p}<0.001 & \mathbf{p}<0.001 & \mathbf{p}<0.001
\end{array} \\
& \chi^{2}(4)=3.47, \quad \chi^{2}(3)=5.63, \quad \chi^{2}(1)=15.0, \\
& \mathrm{p}=0.48 \quad \mathrm{p}=0.13 \quad \mathrm{p}<0.001 \\
& \chi^{2}(4)=35.1, \quad \chi^{2}(3)=9.26 \quad \chi^{2}(1)=0.021 \text {, } \\
& \begin{array}{lll}
\mathbf{p}<0.001 & \mathrm{p}=0.03 \quad \mathrm{p}=0.88
\end{array} \\
& \chi^{2}(4)=51.0, \quad \chi^{2}(3)=33.8, \quad \chi^{2}(1)=19.7, \\
& \mathbf{p}<0.001 \quad \mathbf{p}<0.001 \quad \mathbf{p}<0.001 \\
& \chi^{2}(4)=83.9, \quad \chi^{2}(3)=49.3, \quad \chi^{2}(1)=1.51, \\
& \mathbf{p}<0.001 \quad \mathbf{p}<0.001 \quad \mathrm{p}=0.22 \\
& \chi^{2}(4)=8.78, \quad \chi^{2}(3)=1.82, \quad \chi^{2}(1)=1.52, \\
& \mathrm{p}=0.07 \quad \mathrm{p}=0.61 \quad \mathrm{p}=0.22
\end{aligned}
$$

220 See Figure 2C

$$
\begin{array}{lll}
\chi^{2}(4)=13.0, & \chi^{2}(3)=15.1, & \chi^{2}(1)=36.0, \\
p=0.01 & \mathbf{p}=0.002 & \mathbf{p}<0.001 \\
\chi^{2}(4)=0.493, & \chi^{2}(3)=2.58, & \chi^{2}(1)=3.59, \\
p=0.97 & p=0.46 & p=0.06 \\
\chi^{2}(4)=11.8, & \chi^{2}(3)=6.55, & \chi^{2}(1)=19.0, \\
p=0.02 & p=0.09 & \mathbf{p}<0.001 \\
\chi^{2}(4)=6.47, & \chi^{2}(3)=3.38, & \chi^{2}(1)=17.7, \\
p=0.17 & p=0.34 & \mathbf{p}<0.001
\end{array}
$$




\section{Other}

Travel time for indirect benefit study: Maximum time willing to travel from home for study with direct benefit (0.5-more than $2 \mathrm{hrs})$

Travel time for direct benefit study: Maximum time willing to travel from home for study without direct benefit (0.5-more than $2 \mathrm{hrs}$ )

Overnight trip: Willingness to make overnight or extended trip for research study

Travel reimbursement for a local study: Importance that cost of travel to local study is reimbursed (0-100)

Travel reimbursement for a distant study: Importance that cost of travel to distant study is reimbursed (0-100)

$$
\begin{array}{lll}
\chi^{2}(4)=11.0, & \chi^{2}(3)=7.62, & \chi^{2}(1)=11.0 \\
\mathrm{p}=0.03 & \mathrm{p}=0.06 & \mathrm{p}=0.001
\end{array}
$$

220

$$
4(3)
$$

$3.53(1.40)$

$\chi^{2}(16)=19.1$,

$\chi^{2}(12)=12.1$,

$\chi^{2}(4)=9.00$,

$\mathrm{p}=0.26$

$\mathrm{p}=0.44$

$\mathrm{p}=0.06$
$5(2)$

$4.13(1.19)$ $\chi^{2}(16)=17.3$, $\mathrm{p}=0.37$ $\chi^{2}(12)=17.1$, $\mathrm{p}=0.15$ $\chi^{2}(4)=7.25$, $\mathrm{p}=0.12$
221

203

205
Yes: 110

No: 19

Maybe: 92

$50(57)$

$49.1(32.8)$

$78(32)$

$75.3(24.3)$ $\chi^{2}(8)=2.09$,

$$
\mathrm{p}=0.98
$$

$\chi^{2}(6)=3.87$,

$\mathrm{p}=0.69$

$H(4)=6.87$,

$\mathrm{p}=0.14$

$H(3)=1.22$,

$\mathrm{p}=0.75$

$\mathrm{H}(4)=3.11$, $\mathrm{p}=0.54$
$H(3)=0.431$,

$\mathrm{p}=0.93$ $\chi^{2}(2)=0.574$, $\mathrm{p}=0.75$

$H(1)=7.31$, $\mathrm{p}=0.007$

$H(1)=0.163$, $\mathrm{p}=0.69$

\section{Study-specific Elements}

Locations: Preferred study location

Current clinic

New clinic

Park

Lab

\begin{tabular}{|c|c|c|}
\hline $\begin{array}{l}\chi^{2}(4)=2.28 \\
p=0.68\end{array}$ & $\begin{array}{l}\chi^{2}(3)=1.79 \\
p=0.62\end{array}$ & $\begin{array}{l}\chi^{2}(1)=4.88, \\
\mathbf{p}=0.03\end{array}$ \\
\hline $\begin{array}{l}\chi^{2}(4)=5.01 \\
p=0.29\end{array}$ & $\begin{array}{l}\chi^{2}(3)=13.8 \\
\mathbf{p}=0.003\end{array}$ & $\begin{array}{l}\chi^{2}(1)=0.006, \\
p=0.94\end{array}$ \\
\hline $\begin{array}{l}\chi^{2}(4)=21.6 \\
\mathbf{p}<0.001\end{array}$ & $\begin{array}{l}\chi^{2}(3)=10.7 \\
\mathbf{p}=0.01\end{array}$ & $\begin{array}{l}\chi^{2}(1)=5.67 \\
\mathbf{p}=0.02\end{array}$ \\
\hline $\begin{array}{l}\chi^{2}(4)=26.3 \\
p=0.58\end{array}$ & $\begin{array}{l}\chi^{2}(3)=13.6 \\
\mathbf{p}=0.003\end{array}$ & $\begin{array}{l}\chi^{2}(1)=0.304, \\
p=0.58\end{array}$ \\
\hline $\begin{array}{l}\chi^{2}(4)=2.53 \\
p=0.64\end{array}$ & $\begin{array}{l}\chi^{2}(3)=4.79 \\
p=0.19\end{array}$ & $\begin{array}{l}\chi^{2}(1)=15.0, \\
\mathbf{p}<0.001\end{array}$ \\
\hline $\begin{array}{l}\chi^{2}(4)=7.42 \\
p=0.12\end{array}$ & $\begin{array}{l}\chi^{2}(3)=0.369 \\
p=0.95\end{array}$ & $\begin{array}{l}\chi^{2}(1)=1.80, \\
p=0.18\end{array}$ \\
\hline $\begin{array}{l}\chi^{2}(4)=1.86 \\
p=0.76\end{array}$ & $\begin{array}{l}\chi^{2}(3)=3.65, \\
p=0.30\end{array}$ & $\begin{array}{l}\chi^{2}(1)=1.14 \\
p=0.29\end{array}$ \\
\hline
\end{tabular}

School

Home

Other 
Time of year: Times that would be considered for research

participation

Weekends during school year

Weekdays during school year

Summer break

Spring break

Winter break

Non-attendance school days

Maximum time commitment:

Amount of time in one day that is reasonable to participate in a study (0.5-8 hrs)

Maximum study visits: Maximum number of visits for one study (1-5 visits)

Longitudinal:

Willingness to participate in longitudinal study (Yes or No)

Compensation amount:

Appropriate amount of compensation $(\$ / \mathrm{hr})$
127

See Figure $2 E$

\begin{tabular}{|c|c|c|c|}
\hline & $\begin{array}{l}\chi^{2}(4)=7.46 \\
p=0.11\end{array}$ & $\begin{array}{l}\chi^{2}(3)=7.56, \\
p=0.06\end{array}$ & $\begin{array}{l}\chi^{2}(1)=0.171 \\
p=0.68\end{array}$ \\
\hline & $\begin{array}{l}\chi^{2}(4)=9.23 \\
p=0.06\end{array}$ & $\begin{array}{l}\chi^{2}(3)=4.62, \\
p=0.20\end{array}$ & $\begin{array}{l}\chi^{2}(1)=3.99 \\
\mathbf{p}=0.05\end{array}$ \\
\hline & $\begin{array}{l}\chi^{2}(4)=2.31 \\
p=0.68\end{array}$ & $\begin{array}{l}\chi^{2}(3)=0.546 \\
p=0.91\end{array}$ & $\begin{array}{l}\chi^{2}(1)=14.5 \\
\mathbf{p}<0.001\end{array}$ \\
\hline & $\begin{array}{l}\chi^{2}(4)=3.61 \\
p=0.46\end{array}$ & $\begin{array}{l}\chi^{2}(3)=1.83 \\
p=0.61\end{array}$ & $\begin{array}{l}\chi^{2}(1)=2.59 \\
p=0.11\end{array}$ \\
\hline & $\begin{array}{l}\chi^{2}(4)=1.26 \\
p=0.87\end{array}$ & $\begin{array}{l}\chi^{2}(3)=2.89 \\
p=0.41\end{array}$ & $\begin{array}{l}\chi^{2}(1)=9.96 \\
\mathbf{p}=0.002\end{array}$ \\
\hline & $\begin{array}{l}\chi^{2}(3)=5.73 \\
p=0.22\end{array}$ & $\begin{array}{l}\chi^{2}(3)=5.38 \\
p=0.15\end{array}$ & $\begin{array}{l}\chi^{2}(1)=9.65 \\
\mathbf{p}=0.002\end{array}$ \\
\hline $\begin{array}{c}4(3) \\
3.83(1.16) \\
\text { See Figure } 2 F\end{array}$ & $\begin{array}{l}H(4)=7.57 \\
p=0.11\end{array}$ & $\begin{array}{l}H(3)=14.0 \\
p=0.003\end{array}$ & $\begin{array}{l}H(1)=9.41 \\
p=0.002\end{array}$ \\
\hline $\begin{array}{c}5(2) \\
4.28(1.10)\end{array}$ & $\begin{array}{l}H(4)=2.66 \\
p=0.62\end{array}$ & $\begin{array}{l}H(3)=1.38 \\
p=0.71\end{array}$ & $\begin{array}{l}H(1)=1.61 \\
p=0.21\end{array}$ \\
\hline $\begin{array}{l}\text { Yes: } 210 \\
\text { No: } 10\end{array}$ & $\begin{array}{l}\chi^{2}(4)=6.09 \\
p=0.19\end{array}$ & $\begin{array}{l}\chi^{2}(3)=0.400 \\
p=0.94\end{array}$ & $\begin{array}{l}\chi^{2}(1)=1.75 \\
p=0.19\end{array}$ \\
\hline $\begin{array}{c}15(10) \\
16.7(12.3)\end{array}$ & $\begin{array}{l}H(4)=2.17 \\
p=0.71\end{array}$ & $\begin{array}{l}H(3)=1.51 \\
p=0.68\end{array}$ & $\begin{array}{l}H(1)=0.006 \\
p=0.94\end{array}$ \\
\hline
\end{tabular}

\section{Travel Needs}

Most respondents needed time off work (62.7\%) but did not need additional arrangements for childcare to engage in research $(66.1 \%)$. There was a significant main effect of responder type on the latter, where caregivers of minors with CP needed childcare more than adults with CP. When leaving their homes, the majority of 
medRxiv preprint doi: https://doi.org/10.1101/2021.07.28.21261262; this version posted July 31, 2021. The copyright holder for this preprint (which was not certified by peer review) is the author/funder, who has granted medRxiv a license to display the preprint in perpetuity. It is made available under a CC-BY-NC-ND 4.0 International license .

respondents had additional travel needs such as time (59.2\%), transportation items such as a wheelchair or stroller (58.0\%), and snacks (52.9\%) (Figure 3A).

Specific additional travel needs varied significantly based on GMFCS level, CP type, and responder type. All significant pairwise comparisons are reported in the supplemental section (S1 Table). There was a significant main effect of GMFCS level on breathing items, seizure items, feeding items, medications, toileting items, and transportation items. In summary, individuals classified as GMFCS level V reported needing these items more to comfortably travel. There was a significant main effect of CP type on time, feeding items, toileting items, and transportation items. In general, individuals affected by quadriplegia reported needing these items more to comfortably travel. Finally, there was a significant main effect of responder type on transition, seizure items, feeding items, snacks, and toileting items. Adults with CP had more concerns about transition to a new environment than caregivers of minors with CP. However, caregivers needed seizure items, feeding items, snacks, and toileting items for their children more than adults with CP did for themselves. 
medRxiv preprint doi: https://doi.org/10.1101/2021.07.28.21261262; this version posted July 31, 2021. The copyright holder for this preprint (which was not certified by peer review) is the author/funder, who has granted medRxiv a license to display the preprint in perpetuity. It is made available under a CC-BY-NC-ND 4.0 International license .

A. Additional considerations for $\mathrm{CP}$ research participants when leaving home

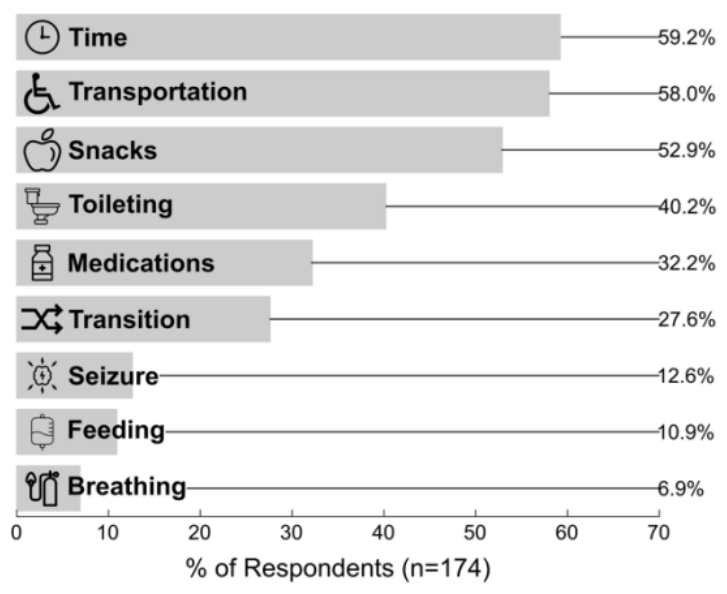

B. Most common transportation methods*

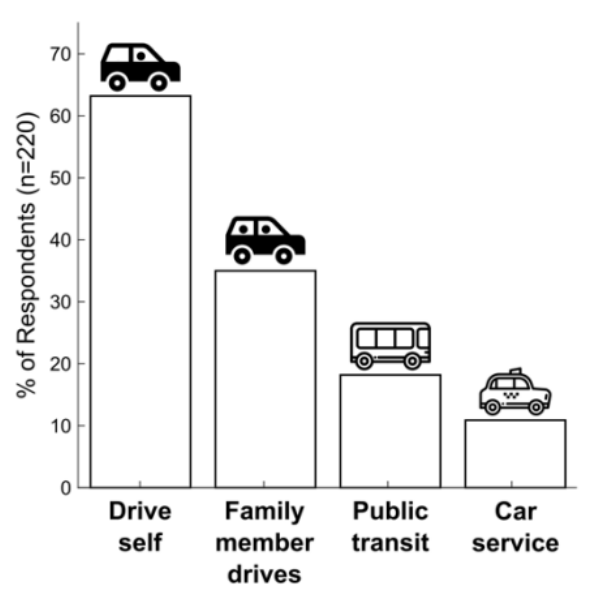

Figure 3: (A) Summary of additional travel needs required to participate in CP research. The most prevalent categories were time, transportation, and snacks. (B) Summary of the most common transportation methods. Driving was the most cited mode of transportation. ${ }^{*} 6.8 \%$ of respondents selected other transportation modes.

\section{Travel Preferences}

The most common mode of transportation was by car, whether the individual drives $(63.2 \%)$ or gets a ride from a family member (35.0\%) (Figure 3B). There was a significant main effect of GMFCS level on transportation methods including drive self, public transit, and other, though there were no significant pairwise comparisons. There was a significant main effect of CP type on drive self, where respondents (both caregivers and adults with CP) affected by hemiplegia preferred to drive themselves more than those affected by diplegia or quadriplegia. There was a significant main effect of responder type on drive self, public transit, ride service, and other. Caregivers of minors with CP preferred to drive themselves more than adults with $\mathrm{CP}$, whereas adults preferred public transit, ride services, or other methods of transportation.

The mean response for travel time for indirect benefit study was 3.53 hours, which was significantly lower $(z=-6.857, p<0.001)$ than the mean response for travel time for 
medRxiv preprint doi: https://doi.org/10.1101/2021.07.28.21261262; this version posted July 31, 2021. The copyright holder for this preprint (which was not certified by peer review) is the author/funder, who has granted medRxiv a license to display the preprint in perpetuity. It is made available under a CC-BY-NC-ND 4.0 International license .

direct benefit study at 4.13 hours. Approximately half $(49.8 \%)$ of respondents were willing to make an overnight trip for research participation. There was a significant main effect of responder type on the importance of travel reimbursement for a local study, where adults with $\mathrm{CP}$ thought reimbursement was more important than caregivers of minors with $\mathrm{CP}$. The overall mean score for this variable (49.1/100) was significantly lower $(z=-9.901, p$ $<0.001$ ) than the mean score for the importance of travel reimbursement for a distant study (75.3/100).

\section{Study-specific Elements}

A current clinic (88.7\%), home (84.7\%), lab (73.0\%), and new clinic (59.9\%) were the most preferred locations for research participation (Figure 4A). There was a significant main effect of GMFCS level on park, where individuals who are GMFCS level I were more likely to select this location than all other levels. There was a significant main effect of CP type on new clinic and lab, where individuals affected by hemiplegia were more likely to select these locations over those affected by quadriplegia. There was a significant main effect of responder type on current clinic, park, and school, where caregivers of minors with $\mathrm{CP}$ were more likely to select these locations over adults with $\mathrm{CP}$.

The majority of respondents were flexible to participate in research at any time of year, except for parents on weekdays during the school year (Figure 4C). There was a significant main effect of responder type on weekdays, where adults reported more availability compared to caregivers. There was also a significant main effect of responder type for summer break, winter break, and other school holidays, where caregivers were more willing to engage in research during these times of year than adults. Respondents indicated a mean compensation amount of $\$ 16.69 /$ hour for participation in research. The 
medRxiv preprint doi: https://doi.org/10.1101/2021.07.28.21261262; this version posted July 31, 2021. The copyright holder for this preprint (which was not certified by peer review) is the author/funder, who has granted medRxiv a license to display the preprint in perpetuity. It is made available under a CC-BY-NC-ND 4.0 International license.

average maximum time commitment was 3.83 hours/day and the average maximum study visits was 4.28 visits. Survival analyses on maximum time commitment yielded significant main effects of CP type (log rank $\left.\chi^{2}(1)=11.9, \mathrm{p}=0.001\right)$, with no significant pairwise comparisons, and responder type $\left(\log\right.$ rank $\left.\chi^{2}(3)=13.4, p=0.004\right)$. Most notably, caregiver interest dropped from $54.1 \%$ to $24.1 \%$ at a maximum time commitment greater than 4 hours (Figure 4B). Finally, the vast majority of respondents were willing to participate in a longitudinal study (95.5\%). 
A. Preferred locations for research participation*

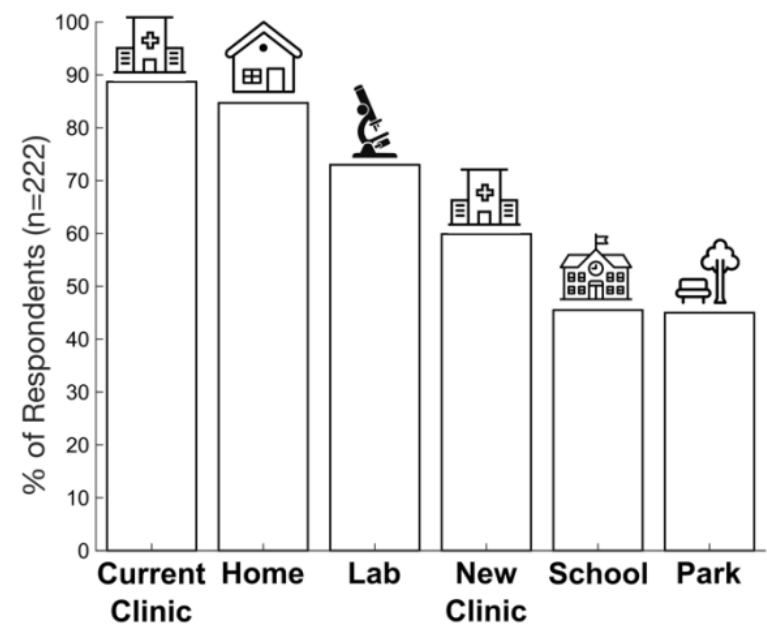

B. Maximum time commitment for study visit

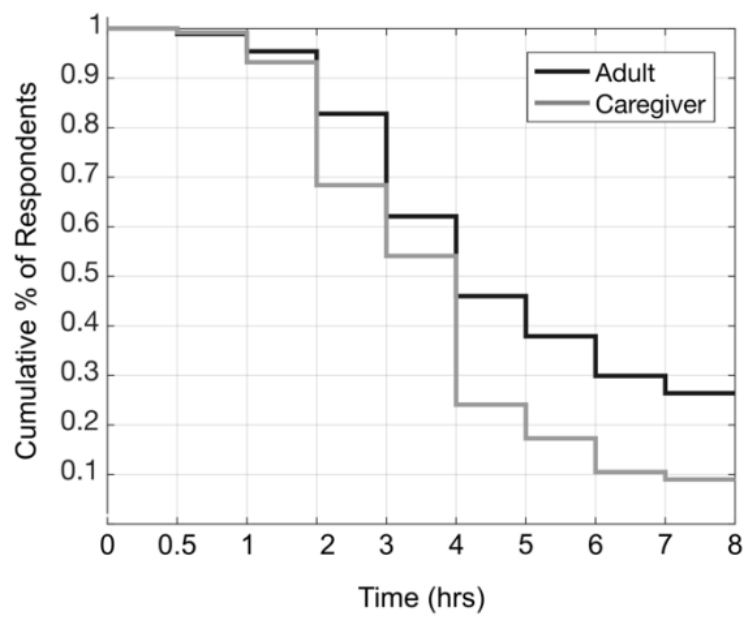

C. Preferred time of year for study participation

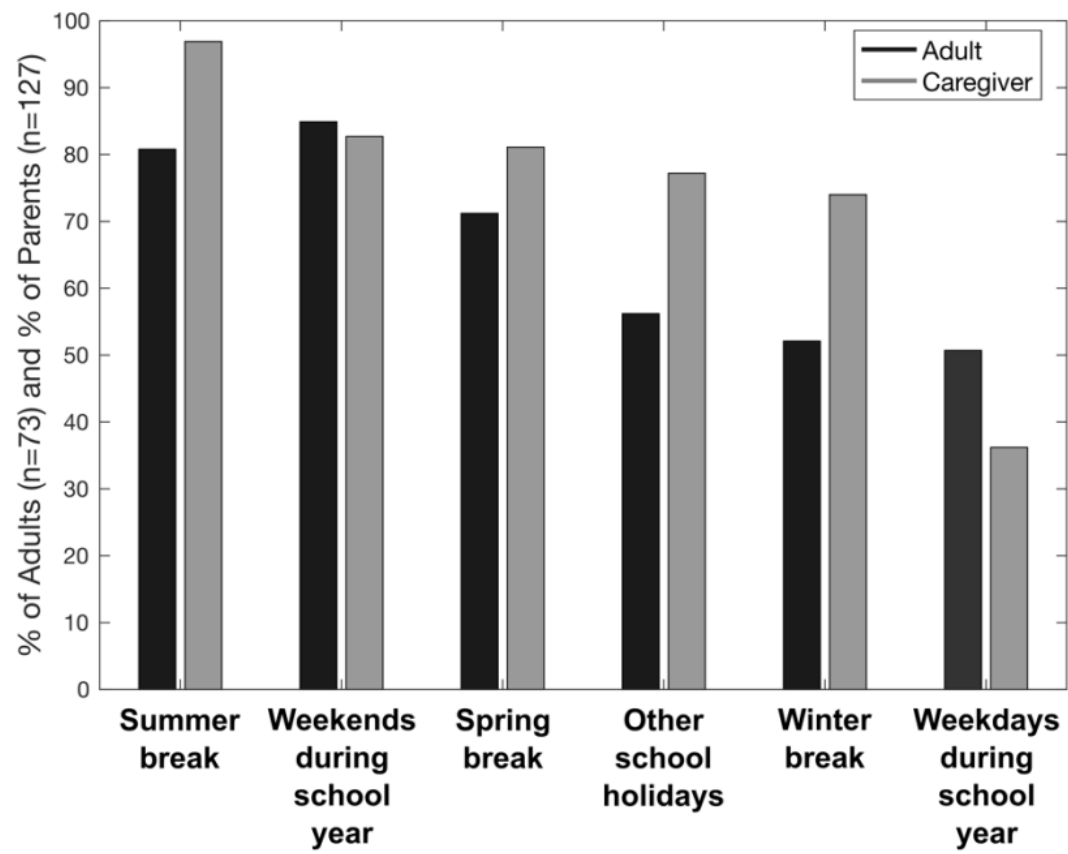

Figure 4: (A) Summary of preferred locations for CP studies. ${ }^{*} 5.4 \%$ of respondents chose other locations. (B) Survival analysis for maximum time commitment by responder type. Less than $30 \%$ of caregivers and adults remain at 4 and 7 hours, respectively. (C) Summary of preferred time of year for participation by responder type.

\section{Discussion}

The purpose of this study was to determine the motivators and barriers involved in the decision to participate in CP research studies. We administered a survey to gain more 
medRxiv preprint doi: https://doi.org/10.1101/2021.07.28.21261262; this version posted July 31, 2021. The copyright holder for this preprint (which was not certified by peer review) is the author/funder, who has granted medRxiv a license to display the preprint in perpetuity. It is made available under a CC-BY-NC-ND 4.0 International license .

insight on stakeholder perspectives, including their personal interest in research, travel needs and preferences for study participation, and study-specific elements. These results can be extrapolated into recommendations for future CP research studies to maximize participant recruitment and expedite new knowledge about CP.

Our study is one of the first to identify the personal and practical factors that influence research participation. Respondents overwhelmingly supported research and valued their own participation in research, in a wide range of topics (Figure 2A). The research areas of interest identified by our survey respondents are largely consistent with previous reports of CP research priorities $[8,16]$. Survey responses indicated that most individuals were motivated to participate by the potential for personal benefit and helping others. Where relevant, these two elements should be highlighted in recruitment materials and results should be disseminated to participants in a format that is best suited to their learning preferences (e.g. a copy of a manuscript or poster, a one-page summary, or a short video). Schedule limitations were the most prevalent barriers to research participation, especially as caregivers of minors with CP were likely to need additional childcare arrangements. Travel limitations were also a highly cited barrier to research participation. To minimize these barriers, researchers should offer flexible study times, particularly during the weekends and summer break, and/or utilize local study locations close to the home or clinics where participants are receiving care.

When scheduling participants with CP for a research study located outside of their home, travel needs for participants should be considered. To accommodate the additional time required for participants and/or their families to reach the study location, researchers should be flexible with appointment times. Indeed, utilizing flexible study protocols has 
medRxiv preprint doi: https://doi.org/10.1101/2021.07.28.21261262; this version posted July 31, 2021. The copyright holder for this preprint (which was not certified by peer review) is the author/funder, who has granted medRxiv a license to display the preprint in perpetuity. It is made available under a CC-BY-NC-ND 4.0 International license .

previously been identified as a recommendation to improve recruitment in CP research [6]. Researchers should also consider having snacks available for participants, particularly for minors with CP. Unsurprisingly, participants classified as GMFCS level V or with a diagnosis of quadriplegia reported requiring more items in order to comfortably travel. Researchers working with these inclusion criteria might consider the home as a study location to mitigate the barrier of travel burden.

Respondents clearly noted the importance of compensation, as providing compensation to participants is consistent with appreciation of their time. In a previous study where caregivers of minors with CP were consulted on the design of randomized control trials, there was a strong preference for coverage of all treatment costs [17]. Caregivers noted that they may not be able to cover the costs themselves, the participants would be offering their time, and the study benefits were unknown [17]. From our survey responses, a minimum compensation of $\$ 15 /$ hour and a maximum time commitment of 4 hours/day were interpreted to be respectful of the time and commitment to research participation.

In addition to direct payment, compensation for other costs associated with travel should be considered. If a study session requires a longer duration, additional compensation can include paying for a meal. Because most respondents were willing to travel long distances for studies with and without direct benefit, researchers should also consider offering travel reimbursement. This is especially important because travel limitations were a highly cited barrier to research participation. As the most preferred mode of transportation involves driving, suggestions for reimbursement include gas and parking. Flexibility around transport mode could also include fare coverage for adult 
medRxiv preprint doi: https://doi.org/10.1101/2021.07.28.21261262; this version posted July 31, 2021. The copyright holder for this preprint (which was not certified by peer review) is the author/funder, who has granted medRxiv a license to display the preprint in perpetuity. It is made available under a CC-BY-NC-ND 4.0 International license .

participants who prefer public transit or ride services. For participants willing to make an overnight trip for a research study, researchers should consider compensating for lodging and overnight parking.

Our survey-based recommendations are centered around maximizing stakeholder participation in CP research studies. One limitation of our sample was that survey respondents were self-selected and may be biased towards research participation. Their responses may inflate measures of research importance and resource allocation (e.g. time and money), while underestimating obstacles to research participation. However, these individuals may also be more likely to respond to participant requests and therefore would be more representative of future study samples. Another limitation is that survey responses were self-reported at one time point. Future research should determine whether attitudes towards research shift over time or are dependent on the depth of previous research experience. Finally, researchers should be informed about and supported in the engagement of the community. This can span a spectrum of involvement, such as one-time consultations to provide feedback on study-specific elements, formation of Community Advisory Boards, or the inclusion of community stakeholders as project investigators.

We assessed the motivators and barriers to research participation from the perspectives of caregivers of minors with $\mathrm{CP}$ and adults with $\mathrm{CP}$. By identifying these stakeholder attitudes and utilizing the information to design study protocols, individuals with CP and their families become true partners in the research that aims to benefit them. Researchers can best accommodate the needs of participants with CP by opting for flexible study locations, scheduling, and compensation options. We recognize that this 
medRxiv preprint doi: https://doi.org/10.1101/2021.07.28.21261262; this version posted July 31, 2021. The copyright holder for this preprint (which was not certified by peer review) is the author/funder, who has granted medRxiv a license to display the preprint in perpetuity. It is made available under a CC-BY-NC-ND 4.0 International license.

will not be feasible for all studies but encourage researchers to consider even the smallest gestures to reduce the burden of participation.

\section{Acknowledgements}

The authors would like to thank Divya Joshi, Nayo Hill, and Heidi Roth for their help in the design of survey questions and recruitment of participants. 
medRxiv preprint doi: https://doi.org/10.1101/2021.07.28.21261262; this version posted July 31, 2021. The copyright holder for this preprint

(which was not certified by peer review) is the author/funder, who has granted medRxiv a license to display the preprint in perpetuity.

It is made available under a CC-BY-NC-ND 4.0 International license .

\section{References}

1. Rosenbaum P, Paneth N, Leviton A, Goldstein M, Bax M, Damiano D, et al. A report: the definition and classification of cerebral palsy April 2006. Dev Med Child Neurol Suppl. 2007;109:8-14.

2. Wimalasundera N, Stevenson VL. Cerebral palsy. Pract Neurol [Internet]. 2016 Jun 1 [cited 2021 Mar 18];16(3):184-94. Available from: http://pn.bmj.com/

3. Odding E, Roebroeck ME, Stam HJ. The epidemiology of cerebral palsy: Incidence, impairments and risk factors. Disabil Rehabil [Internet]. 2006 Feb [cited 2021 Jun 23];28(4):183-91. Available from:

https://www.tandfonline.com/doi/abs/10.1080/09638280500158422

4. Blair E, Watson L. Epidemiology of cerebral palsy [Internet]. Vol. 11, Seminars in Fetal and Neonatal Medicine. Elsevier; 2006 [cited 2021 Jun 23]. p. 117-25. Available from: http://www.sfnmjournal.com/article/S1744165X05000910/fulltext

5. Korzeniewski SJ, Slaughter J, Lenski M, Haak P, Paneth N. The complex aetiology of cerebral palsy [Internet]. Vol. 14, Nature Reviews Neurology. Nature Publishing Group; 2018 [cited 2021 Jun 23]. p. 528-43. Available from: https://doi.org/10.1038/

6. Beckers LWME, Rameckers EAA, Smeets RJEM, Van Der Burg JJW, Aarts PBM, Schnackers MLAP, et al. Barriers to recruitment of children with cerebral palsy in a trial of home-based training. 2019 [cited 2021 Jan 7]; Available from: https://doi.org/10.1016/j.conctc.2019.100371

7. Wu YW, Mehravari AS, Numis AL, Gross P. Cerebral palsy research funding from the National Institutes of Health, 2001 to 2013. Dev Med Child Neurol [Internet]. 2015 Oct 1 [cited 2021 Jan 7];57(10):936-41. Available from: http://doi.wiley.com/10.1111/dmcn.12789

8. Gross PH, Bailes AF, Horn SD, Hurvitz EA, Kean J, Shusterman M. Setting a patient-centered research agenda for cerebral palsy: a participatory action research initiative. Dev Med Child Neurol. 2018 Dec 1;60(12):1278-84.

9. Lungu C, Hirtz D, Damiano D, Gross P, Mink JW. Report of a workshop on research gaps in the treatment of cerebral palsy. In: Neurology [Internet]. Lippincott Williams and Wilkins; 2016 [cited 2021 Jan 7]. p. 1293-8. Available 
medRxiv preprint doi: https://doi.org/10.1101/2021.07.28.21261262; this version posted July 31, 2021. The copyright holder for this preprint

(which was not certified by peer review) is the author/funder, who has granted medRxiv a license to display the preprint in perpetuity. It is made available under a CC-BY-NC-ND 4.0 International license .

from: https://n.neurology.org/content/87/12/1293

10. Mcintyre S, Novak I, Cusick A. Consensus research priorities for cerebral palsy: A Delphi survey of consumers, researchers, and clinicians. Dev Med Child Neurol [Internet]. 2010 Mar 1 [cited 2021 Jun 23];52(3):270-5. Available from: https://onlinelibrary.wiley.com/doi/full/10.1111/j.1469-8749.2009.03358.x

11. Hurley DS, Sukal-Moulton T, Msall ME, Gaebler-Spira D, Krosschell KJ, Dewald JP. The Cerebral Palsy Research Registry: Development and Progress Toward National Collaboration in the United States. J Child Neurol [Internet]. 2011 [cited 2019 Mar 3];26(12):1534-41. Available from: http://jcn.sagepub.com

12. Hurvitz EA, Gross PH, Gannotti ME, Bailes AF, Horn SD. Registry-based Research in Cerebral Palsy The Cerebral Palsy Research Network. Phys Med Rehabil Clin N Am [Internet]. 2020 [cited 2021 Jan 7];31:185-94. Available from: https://doi.org/10.1016/j.pmr.2019.09.005

13. Joshi D, Hill N, Hruby A, Viswanathan S, Ingo C, Roth $\mathrm{H}$, et al. Stakeholder Perspectives on Engaging with Cerebral Palsy Research Studies Following Onset of COVID-19 in the United States. Arch Phys Med Rehabil [Internet]. 2021 Mar 10 [cited 2021 Mar 18]; Available from:

http://www.ncbi.nlm.nih.gov/pubmed/33713698

14. Howden LM, Meyer JA. Age and Sex Composition: 2010 [Internet]. 2011 [cited 2021 Mar 18]. Available from: www.census.gov/population

15. Humes KR, Jones NA, Ramirez RR. Overview of Race and Hispanic Origin: 2010 2010 Census Briefs [Internet]. 2010 [cited 2021 Mar 18]. Available from: www.whitehouse.gov/omb

16. Shriver EK. NINDS/NICHD Strategic Plan for Cerebral Palsy Research [Internet]. 2017 [cited $2021 \mathrm{Feb} 22$ ]. Available from: https://www.cdc.gov/ncbddd/cp/data.html.

17. Edwards V, Wyatt K, Logan S, Britten N. Consulting parents about the design of a randomized controlled trial of osteopathy for children with cerebral palsy. Heal Expect [Internet]. $2011 \mathrm{Dec}$ [cited $2021 \mathrm{Mar}$ 18];14(4):429-38. Available from: /pmc/articles/PMC5060591/ 